\title{
Computer simulation and theoretical results for a polar-polarizable fluid
}

\author{
by J. M. CAILlol, D. LEVEsQUE and J. J. WEIS \\ Laboratoire de Physique Théorique et Hautes Energies†, \\ Université de Paris-Sud, 91405 Orsay, France \\ and P. G. KUSALIK and G. N. PATEY \\ Department of Chemistry, University of British Columbia, \\ Vancouver, British Columbia, Canada V6T 1 Y6
}

(Received 17 September 1984 ; accepted 17 December 1984)

\begin{abstract}
In this paper we present molecular dynamics (MD) results for a fluid of polarizable Lennard-Jones particles with permanent dipole and quadrupole moments. Detailed comparisons are made with theoretical results obtained using a previously developed self-consistent mean field (SCMF) approximation together with the $\mathrm{LHNC}$ and $\mathrm{QHNC}$ integral equation theories. The $\mathrm{SCMF}$ approximation is found to give a reasonably accurate description of the polarizable system. The SCMF/QHNC and SCMF/LHNC calculations both give values for the dielectric constant and average dipole moment which are in good agreement with the MD simulations. The SCMF/QHNC theory gives better results for the pair correlation function and for some thermodynamic properties.
\end{abstract}

\section{InTRODUCTION}

In a recent article [1] we have presented molecular dynamics (MD) simulation results for a non-polarizable generalized Stockmayer fluid consisting of Lennard-Jones (LJ) spheres with embedded point dipoles and tetrahedral quadrupoles. These calculations were used to test the linearized and quadratic hypernetted-chain (LHNC and QHNC) approximations for the non-polarizable case. However, a previous approximate theoretical treatment [2] has indicated that if water-like parameters are used in a polarizable dipole-tetrahedral quadrupole model, the many-body polarization effects play an important role and are particularly significant in the determination of the dielectric constant. The principal purpose of the present work is to test and evaluate these theoretical predictions by carrying out computer simulations of a fully polarizable model.

In this paper we report MD calculations for a polarizable generalized Stockmayer fluid and make detailed comparisons with the results given by the selfconsistent mean field (SCMF) approximation described in [2]. For purely dipolar fluids the SCMF approximation is equivalent to the renormalized 1 $\mathrm{R}$ theory first derived by Wertheim [3]. Also we note that the renormalized theory 
has been recently extended [4] in order to calculate the thermodynamic properties of polarizable dipole-quadrupole fluids.

The SCMF theory, as applied in [2], reduces the many-body energy of a polarizable system to a sum over pairwise additive interactions characterized by an effective permanent dipole moment. This effective dipole moment depends in a non-trivial way upon the polarizability, the permanent multipole moments, and other properties of the model, as well as upon the state parameters of the system. Within the SCMF approximation the effective system has the same structural and dielectric properties as the true polarizable fluid. However, we emphasize that the energy of the effective system cannot be directly compared with that of the polarizable fluid. In order to obtain the energy of the polarizable fluid, the energy required to polarize the particles must be taken into account. This leads to a correction of the effective system energy as shown in [2].

In order to apply the SCMF theory, it is necessary to calculate the properties of the system defined by the effective pair potential. In the present calculations as in previous work $[1,2]$ this is done using the LHNC and QHNC theories.

The remainder of this paper is divided into four parts. The details of the model and computer calculations are given in $\S 2$, the SCMF theory is briefly described in $\S 3$, the results are discussed in $\S 4$, and our conclusions are summarized in $\S 5$.

\section{The model and COMPUter Simulation}

The MD simulations were carried out with $N=256$ particles enclosed in a cubical box. Electrostatically, each particle is characterized by a permanent dipole moment, $\mu$, a quadrupole moment, $Q_{\mathrm{T}}$, and a constant point polarizability, $\alpha$. If the set of orthogonal unit vectors $\left(\hat{\mathbf{x}}_{i}, \hat{\mathbf{y}}_{i}, \hat{\mathbf{z}}_{i}\right)$ define a molecule-fixed coordinate system then each particle has an associated dipole vector and quadrupole tensor given by

and

$$
\boldsymbol{\mu}_{i}=\mu \hat{\mathbf{z}}_{i}
$$

$$
\mathbf{Q}_{i}=Q_{T}\left(\hat{\mathbf{x}}_{i} \hat{\mathbf{x}}_{i}-\hat{\mathbf{y}}_{i} \hat{\mathbf{y}}_{i}\right),
$$

respectively.

The total instantaneous configurational energy of the system can be written in the form [2]

$$
\begin{aligned}
U= & \frac{1}{2} \sum_{i \neq j} u_{\mathrm{LJ}}(r)-\frac{1}{2} \sum_{i} \mathbf{m}_{i} \cdot \mathbf{E}_{i}^{(m)}-\frac{1}{2} \sum_{i} \mathbf{m}_{i} \cdot \mathbf{E}_{i}^{(\mathbf{Q})} \\
& -\frac{1}{2} \sum_{i \neq j} \frac{1}{9} \mathbf{Q}_{i}: \mathbf{T}_{i j}^{(4)}: \mathbf{Q}_{j}+\frac{1}{2} \sum_{i} \mathbf{p}_{i} \cdot\left(\mathbf{E}_{i}^{(m)}+\mathbf{E}_{i}^{(\mathrm{Q})}\right) \\
\equiv & U_{\mathrm{LJ}}+U_{\mathrm{DD}}+U_{\mathrm{DQ}}+U_{\mathrm{QQ}}+U_{\mathrm{pol}},
\end{aligned}
$$

where

$$
\begin{aligned}
u_{\mathrm{LJ}}(r) & =4 \varepsilon_{\mathrm{LJ}}\left[\left(\frac{\sigma}{r}\right)^{12}-\left(\frac{\sigma}{r}\right)^{6}\right], \\
\mathbf{T}_{i j}^{(n)} & =-\nabla_{i}^{(n)} \frac{1}{\left|\mathbf{r}_{i}-\mathbf{r}_{j}\right|},
\end{aligned}
$$


and $\mathbf{m}_{i}, \boldsymbol{\mu}_{i}$ and $\mathbf{p}_{i}$ denote the total, permanent, and induced dipole moments, respectively. The last term in equation $(2 a), U_{\text {pol }}$, is the energy of polarization for spherical particles. In equation $(2 a) \mathbf{E}_{i}^{(m)}$ and $\mathbf{E}_{i}^{(\mathrm{Q})}$ represent the local electric fields at particle $i$ due to the total dipole and quadrupole moments, respectively, of all other particles in the system. These are defined by

$$
\begin{aligned}
& \mathbf{E}_{i}^{(m)}=-\sum_{j \neq i} \mathbf{T}_{i j}^{(2)} \cdot \mathbf{m}_{j}, \\
& \mathbf{E}_{i}^{(\mathrm{Q})}=\frac{1}{3} \sum_{j \neq i} \mathbf{T}_{i j}^{(3)}: \mathbf{Q}_{j}
\end{aligned}
$$

where $\mathbf{T}_{i j}^{(n)}$ is given by equation $(2 c)$. The total dipole moment, $\mathbf{m}_{i}$, is given by

$$
\mathbf{m}_{i}=\boldsymbol{\mu}_{i}+\mathbf{p}_{i}
$$

and the induced moment

$$
\mathbf{p}_{i}=\alpha\left(\mathbf{E}_{i}^{(m)}+\mathbf{E}_{i}^{(\mathrm{Q})}\right) .
$$

The molecular dynamics simulation of the polarizable fluid was carried out in a manner similar to that previously applied [5-10] to polar-polarizable systems. The electric field $\mathbf{E}_{i}^{(m)}$ is obtained by solving equations $(3 a)-(4 b)$ iteratively. For the value of $\alpha$ considered here four iterations were sufficient if the first estimate of $\mathbf{m}_{j}$ is taken to be the value at the previous time step. The remaining computational details are as described in [1] for the non-polarizable case. In particular, the long-range character of the multipolar interactions is taken into account by using Ewald boundary conditions. This amounts to replacing $1 /\left|\mathbf{r}_{j i}\right|$ in equation (2c) with

$$
\begin{aligned}
& f\left(\mathbf{r}_{j i}\right)=\frac{1}{L} \sum_{\mathbf{n}} \frac{\operatorname{erfc}\left(\eta\left|\mathbf{r}_{j i} / L+\mathbf{n}\right|\right)}{\left|\mathbf{r}_{j i} / L+\mathbf{n}\right|} \\
& \quad+\frac{1}{\pi L} \sum_{\mathbf{n} \neq 0} \frac{1}{|\mathbf{n}|^{2}} \exp \left(-\pi^{2}|\mathbf{n}|^{2} / \eta^{2}+2 \pi i \mathbf{n} \cdot \mathbf{r}_{j i} / L\right),
\end{aligned}
$$

where the sum on $\mathbf{n}$ is over all integer coordinates, $\mathbf{n}=(k, l, m)$, and $L$ is the length of the cubic simulation cell. In the present computations the parameter $\eta$ was adjusted such that $\eta L=5 \cdot 76$. The interactions arising from the first term in equation (5) were truncated at $L / 2$ and the sum in the second term included all vectors such that $|\mathbf{n}| \leqslant 5$. The equations of motion were solved with a time step, $\Delta t=0 \cdot 002 \tau_{0}$, where $\tau_{0}=\sqrt{ }\left(m \sigma^{2} / \varepsilon_{\mathrm{LJ}}\right)$, and the moment of inertia was chosen to be 1 in reduced units.

The dielectric constant was obtained using the formula $[6,11-13]$

$$
\frac{\varepsilon-1}{3}=y \frac{\left\langle M^{2}\right\rangle}{N \mu^{2}}+\frac{\varepsilon_{\infty}-1}{3},
$$

where $\left\langle M^{2}\right\rangle$ is the mean square moment of the sample, $\varepsilon_{\infty}$ is the high frequency dielectric constant, and $y=4 \pi \rho \mu^{2} / 9 k T$ with $\rho$ being the number density. We note that Neumann and Steinhauser [11] have recently pointed out that equation (6) is strictly applicable to the computer simulation situation only when the integral

$$
q=\int_{0}^{R_{\mathrm{c}}} 4 \pi r^{2}\left(\eta / \pi^{1 / 2}\right)^{3} \exp \left(-\eta^{2} r^{2}\right) d r
$$


is equal to 1 . In equation (7) $R_{\mathrm{c}}$ is the radius at which the real space sum in equation (5) is truncated. In the present calculations $R_{\mathrm{c}}=L / 2, \eta L=5.76$ and equation (7) gives $q=0.9991$ which is very close to 1 . Thus for our simulations the $q$-dependent correction given by Neumann and Steinhauser [11] makes a contribution to $\varepsilon$ which is negligibly small (i.e. $<1$ per cent) compared with the uncertainty (i.e. $\sim 10$ per cent) in the MD estimation. This is also true of the results described in [1].

The contribution of the term $\left(\varepsilon_{\infty}-1\right) / 3$ in equation (6) to $\varepsilon$ could be found exactly [14] but for the present calculations the Clausius-Mossotti relationship

$$
\frac{\varepsilon_{\infty}-1}{\varepsilon_{\infty}+2}=\frac{4 \pi}{3} \rho \alpha
$$

is sufficiently accurate. For the system we consider $\rho^{*}=\rho \sigma^{3}=0 \cdot 8, \alpha^{*}=\alpha / \sigma^{3}=$ $0 \cdot 05$ and equation (8) gives $\left(\varepsilon_{\infty}-1\right) / 3=0 \cdot 2013$. The contribution of this term to $\varepsilon$ for our model is only $\sim 2.3$ per cent which is much smaller than the statistical error. Thus small corrections to equation (8) are of no importance in the present work.

It can be shown $[5,7]$ that for a fluid of polarizable particles the pressure does not depend directly upon the polarization energy denoted by $U_{\text {pol }}$ in equation (2a). Thus the virial expression for the compressibility factor, $\beta(P / \rho)$, is analogous to that for non-polarizable systems [1]. Explicitly one has

$$
\beta \frac{P}{\rho}=\left(\beta \frac{P}{\rho}\right)_{\mathrm{LJ}}+\beta \frac{\left\langle U_{\mathrm{DD}}\right\rangle}{N}+\frac{4}{3} \beta \frac{\left\langle U_{\mathrm{DQ}}\right\rangle}{N}+\frac{5}{3} \beta \frac{\left\langle U_{\mathrm{QQ}}\right\rangle}{N},
$$

where $U_{\mathrm{DD}}, U_{\mathrm{DQ}}$ and $U_{\mathrm{QQ}}$ are as defined in equation ( $2 a$ ), the angular brackets denote average values and $\beta=1 / k T$. The first term in equation $(9 a)$ is the contribution from the LJ interaction and can be written in the form

$$
\left(\beta \frac{P}{\rho}\right)_{L J}=1-\frac{2 \pi}{3} \beta \rho \int_{0}^{\infty} r^{3} \frac{\partial u_{L J}(r)}{\partial r} g^{000}(r) d r
$$

where $g^{000}(r)$ is the radial distribution function.

In addition to the properties discussed above we also examine the pair correlation function $h(12)$. For the present model it is useful to expand $h(12)$ in the form $[1,2]$

$$
\begin{aligned}
h(12)= & h^{000}(r)+h^{110}(r) \Phi^{110}(12)+h^{112}(r) \Phi^{112}(12)+h^{121}(r) \boldsymbol{\Phi}^{121}(12) \\
& +h^{211}(r) \boldsymbol{\Phi}^{211}(12)+h^{123}(r) \boldsymbol{\Phi}^{123}(12)+h^{213}(r) \boldsymbol{\Phi}^{213}(12) \\
& +h^{220}(r) \boldsymbol{\Phi}^{220}(12)+h^{222}(r) \boldsymbol{\Phi}^{222}(12)+h^{224}(r) \boldsymbol{\Phi}^{224}(12)+\ldots,
\end{aligned}
$$

where the angle-dependent functions, $\boldsymbol{\Phi}^{m n l}(12)$, and the coefficients, $h^{m n l}(r)$, are as defined in [1] and [2]. The coefficients $h^{m n l}(r)$ are calculated in the MD simulations and are compared with theoretical results. We note that symmetry requires that $h^{121}(r)=-h^{211}(r)$ and $h^{123}(r)=-h^{213}(r)$. We also emphasize that the thermodynamic properties and dielectric constant of the polarizable fluid are not simply related to $h(12)$ as they are in the non-polarizable case. The properties of the polarizable system will of course depend upon the many-body and not just the pair correlations. 


\section{THE SCMF THEOHY}

The SCMF theory as applied in [2] replaces the polarizable fluid with an effectire system defined by the pair potential

$$
\begin{aligned}
u_{\mathrm{e}}(12)=u_{\mathrm{LJ}}(r)+u^{112}(r) \Phi^{112}(12) & +u^{123}(r) \boldsymbol{\Phi}^{123}(12) \\
& +u^{213}(r) \boldsymbol{\Phi}^{213}(12)+u^{224}(r) \boldsymbol{\Phi}^{224}(12),
\end{aligned}
$$

where

$$
\begin{aligned}
& u^{112}(r)=-m_{\mathrm{e}}^{2} / r^{3}, \\
& u^{123}(r)=-u^{213}(r)=m_{\mathrm{e}} Q_{T} / \sqrt{ } 6 r^{4}, \\
& u^{224}(r)=Q_{T}^{2} / 2 r^{5}
\end{aligned}
$$

and $m_{\mathrm{e}}^{2}=\left\langle m^{2}\right\rangle$ is the mean square dipole moment for a single particle. We note that equation $(11 c)$ represents a slight simplification of the true SCMF theory [2], which actually gives $u^{123}(r)=m^{\prime} Q_{\mathrm{T}}\left\langle\sqrt{ } 6 r^{4}\right.$, where $\mathbf{m}^{\prime}=\langle\mathbf{m}\rangle$ is the mean molecular dipole moment as measured in the molecular coordinate system. For models such as the present one where the difference between $m^{\prime}$ and $m_{\mathrm{e}}$ is small (i.e. $\sim 3.5$ per cent) replacing the true SCMF effective potential with equation (11c) reduces the computational effort and will not significantly alter the theoretical results.

In order to find the value of $m_{\mathrm{e}}$ consistent with a given molecular polarizability one must solve the equations,

$$
\begin{aligned}
\mathbf{m}^{\prime} & =\boldsymbol{\mu}+C\left(m^{\prime}\right) \boldsymbol{\mu} \cdot \boldsymbol{\alpha}^{\prime}, \\
\boldsymbol{\alpha}^{\prime} & =\boldsymbol{\alpha}+C\left(m^{\prime}\right) \boldsymbol{\alpha} \cdot \boldsymbol{\alpha}^{\prime}, \\
m_{\mathrm{e}}^{2} & =\left\langle m^{2}\right\rangle=m^{\prime 2}+3 \alpha^{\prime} k T,
\end{aligned}
$$

where $\alpha$ and $\alpha^{\prime}$ represent the polarizability and renormalized polarizability [2, 3] tensors, respectively. For the present spherically symmetric model these expressions simplify a little since the polarizability tensors are replaced with the scalers

$\alpha=\frac{1}{3} \operatorname{Tr} \alpha$ and $\alpha^{\prime}=\frac{1}{3} \operatorname{Tr} \alpha^{\prime}$. The scalar $C\left(m^{\prime}\right)$ occurring in equations $(12 a)$ and $(12 b)$ is defined by

$$
\left\langle\mathbf{E}^{(m)}\right\rangle+\left\langle\mathbf{E}^{(\mathrm{Q})}\right\rangle=C\left(m^{\prime}\right) \mathbf{m}^{\prime},
$$

where $\left\langle\mathbf{E}^{(m)}\right\rangle$ and $\left\langle\mathbf{E}^{(\mathbb{Q})}\right\rangle$ are the average total dipole and quadrupole fields, respectively, acting on a molecule. We note that equations (12) are exact relationships for systems of molecules of $C_{2 v}$ or higher symmetry and hence do not depend upon the SCMF approximation.

For our model the SCMF theory gives [2]

$$
C\left(m^{\prime}\right)=-\frac{2\left\langle U_{\mathrm{DD}}\right\rangle_{\mathrm{e}}}{m_{\mathrm{e}}^{2} N}-\frac{\left\langle U_{\mathrm{DQ}}\right\rangle_{\mathrm{e}}}{N m_{\mathrm{e}} m^{\prime}}
$$

where $\left\langle U_{\mathrm{DD}}\right\rangle_{\mathrm{e}}$ and $\left\langle U_{\mathrm{DQ}}\right\rangle_{\mathrm{e}}$ are the average dipole-dipole and dipole-quadrupole energies for the effective system. In the present work these energies are obtained using the LHNC and QHNC theories as described in [2]. In summary, the SCMF theory is solved when equations $(12 a-c)$ and (13) are simultaneously satisfied, and it is obvious that the results obtained will depend upon the theory applied in order to calculate the properties of the effective system. 
In the SCMF theory the average energy of the polarizable system, $\langle U\rangle$, is given by [2]

$$
\langle U\rangle=\left\langle U_{\mathrm{LJ}}\right\rangle_{\mathrm{e}}+\left\langle U_{\mathrm{QQ}}\right\rangle_{\mathrm{e}}+\frac{\mu m^{\prime}}{m_{\mathrm{e}}^{2}}\left\langle U_{\mathrm{DD}}\right\rangle_{\mathrm{e}}+\frac{\left(m^{\prime}+\mu\right)}{2 m_{\mathrm{e}}}\left\langle U_{\mathrm{DQ}}\right\rangle_{\mathrm{e}}
$$

and the compressibility factor is

$$
\beta \frac{P}{\rho}=\left(\frac{\beta P}{\rho}\right)_{L J, \mathrm{e}}+\beta \frac{\left\langle U_{\mathrm{DDD}}\right\rangle_{\mathrm{e}}}{N}+\frac{4}{3} \beta \frac{\left\langle U_{\mathrm{DQ}}\right\rangle_{\mathrm{e}}}{N}+\frac{5}{3} \beta \frac{\left\langle U_{\mathrm{QQ}}\right\rangle_{\mathrm{e}}}{N} .
$$

The dielectric constant of the polarizable fluid is identical to that of the effective system and is given by the formula $[2,3]$

$$
\frac{(\varepsilon-1)(2 \varepsilon+1)}{9 \varepsilon}=y_{\mathrm{e}} g
$$

where

$$
g=1+4 \pi \frac{\rho}{3} \int_{0}^{\infty} h_{\mathrm{e}}^{110}(r) r^{2} d r
$$

$y_{\mathrm{e}}=4 \pi \rho m_{\mathrm{e}}^{2} / 9 k T$ and $h_{\mathrm{e}}^{110}(r)$ is the coefficient multiplying $\Phi^{110}(12)$ in the pair correlation function of the effective system.

\section{Results and discussion}

It is convenient to describe our model fluid with the reduced density $\rho^{*}=\rho \sigma^{3}$, the reduced temperature $T^{*}=k T / \varepsilon_{\mathrm{L} J}$, the reduced dipole moment $\left(\mu^{2} / \varepsilon_{\mathrm{LJ}} \sigma^{3}\right)^{1 / 2}$, the reduced quadrupole moment $Q_{T}^{*}=\left(Q_{T}^{2} / \varepsilon_{\mathrm{LJ}} \sigma^{5}\right)^{1 / 2}$ and the reduced polarizability $\alpha^{*}=\alpha / \sigma^{3}$. In addition we shall refer to the reduced quantities $m^{\prime *}=\left(m^{\prime 2} / \varepsilon_{\mathrm{LJ}} \sigma^{3}\right)^{1 / 2}$ and $\alpha^{\prime *}=\alpha^{\prime} / \sigma^{3}$. Our computer simulations were carried out for the polarizable fluid characterized by $\rho^{*}=0.8, T^{*}=1 \cdot 35, \mu^{*}=1 \cdot 5$, $Q_{T}^{*}=0.72$ and $\alpha^{*}=0.05$. In order to clearly show the influence of molecular polarizability for this model we also report results for a system with the same values of $\rho^{*}, T^{*}, \mu^{*}$ and $Q_{T}^{*}$ but with $\alpha^{*}=0 \cdot 0$.

The details of the LHNC and QHNC theories are given in [2]. In the present work the reference versions of these approximations were solved exactly as described in [1]. The MD calculations were carried out on the CRAY-1 computer at the Ecole Polytechnique (Paris). The MD results for $h(12)$ and the thermodynamic properties were obtained with runs of 15000 time steps. The dielectric constant was calculated by averaging over two separate MD runs each of 15000 time steps.

\section{(a) Thermodynamic, dielectric and single particle properties}

MD and theoretical results for the average energies, the compressibility factor, and the dielectric constant are compared in the table for both the polarizable and the non-polarizable fluid. Also for the polarizable system the reduced average single particle dipole moment, $m^{\prime *}$, and the reduced renormalized polarizability, $\alpha^{\prime *}$, are included in table $(a)$. The rather large influence of polarizability upon the properties of the system is obvious if we compare the MD values given in tables $(a)$ and $(b)$. Notably, for the present model polarizability increases the 
A summary of MD and theoretical results for different fluids.

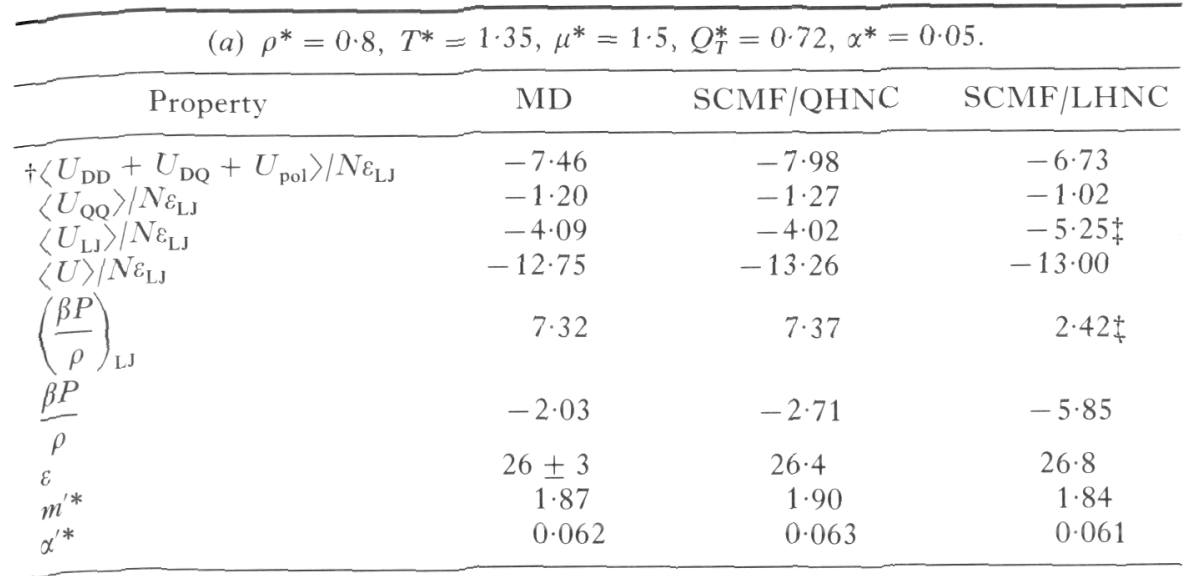

(b) $\rho^{*}=0 \cdot 8, T^{*}=1 \cdot 35, \mu^{*}=1 \cdot 5, Q^{*}=0 \cdot 72, \alpha^{*}=0 \cdot 0$.

\begin{tabular}{lrcc}
\hline Property & MD & QHNC & LHNC \\
\hline$\left\langle U_{\mathrm{DD}}\right\rangle / N \varepsilon_{\mathrm{LJ}}$ & $-2 \cdot 50$ & $-2 \cdot 61$ & $-2 \cdot 47$ \\
$\left\langle U_{\mathrm{DQ}}\right\rangle / N \varepsilon_{\mathrm{LJ}}$ & -2.68 & $-2 \cdot 75$ & $-2 \cdot 50$ \\
$\left\langle U_{\mathrm{QQ}}\right\rangle / N \varepsilon_{\mathrm{LJ}}$ & $-1 \cdot 25$ & $-1 \cdot 31$ & $-1 \cdot 14$ \\
$\left\langle U_{\mathrm{LJ}}\right\rangle / N \varepsilon_{\mathrm{LJ}}$ & -4.57 & $-4 \cdot 61$ & $-5 \cdot 25 \ddagger$ \\
$\langle U\rangle / N \varepsilon_{\mathrm{LJ}}$ & $-11 \cdot 00$ & $-11 \cdot 28$ & $-11 \cdot 36$ \\
$\left(\frac{\beta P}{\rho}\right)_{L J}$ & $5 \cdot 18$ & $5 \cdot 15$ & $2 \cdot 42 \ddagger$ \\
$\frac{\beta P}{\rho}$ & & & $-3 \cdot 29$ \\
$\varepsilon$ & -0.86 & $-1 \cdot 11$ & 12.5 \\
\hline
\end{tabular}

† The MD results for the individual components are $\left\langle U_{\mathrm{DD}}\right\rangle / N \varepsilon_{\mathrm{LJ}}=-5 \cdot 32$, $\left\langle U_{D Q}\right\rangle\left\langle N \varepsilon_{L}=-3.97\right.$ and $\left.\left\langle U_{\text {pol }}\right\rangle\right| N \varepsilon_{L,}=1.83$.

$\$$ These values are MD results for the pure LJ fluid [15].

average dipole moment by $\sim 25$ per cent, the average energy by $\sim 16$ per cent and the static dielectric constant by roughly a factor of two.

It can be seen from table $(a)$ that the agreement between the MD, SCMF/ QHNC and SCMF/LHNC results depends to some extent upon which property one considers. This of course is also true for non-polarizable fluids (cf. table (b) and [1]). From table $(a)$ we see that the MD and SCMF/QHNC results for $\left\langle U_{\mathrm{DD}}+U_{\mathrm{DQ}}+U_{\text {pol }}\right\rangle$ (i.e. in the SCMF theory this is given by the last two terms in equation (14)) differ by $\sim 7$ per cent. The total energies, $\langle U\rangle$, are in better agreement differing by $\sim 4$ per cent. The agreement between the SCMF/LHNC and MD values for $\langle U\rangle$ is quite good, but this is somewhat fortuitous resulting from the cancellation of errors in the different energy components. From table (b) it can be seen that for the non-polarizable fluid the agreement between the QHNC and MD results for $\langle U\rangle$ is a little better than that found for the polarizable case.

The compressibility factors $\beta P / \rho$ given in the table are negative for both the polarizable and non-polarizable fluids. This is not surprising in view of previous results [1] and indicates that these systems are in fact metastable. We note that 


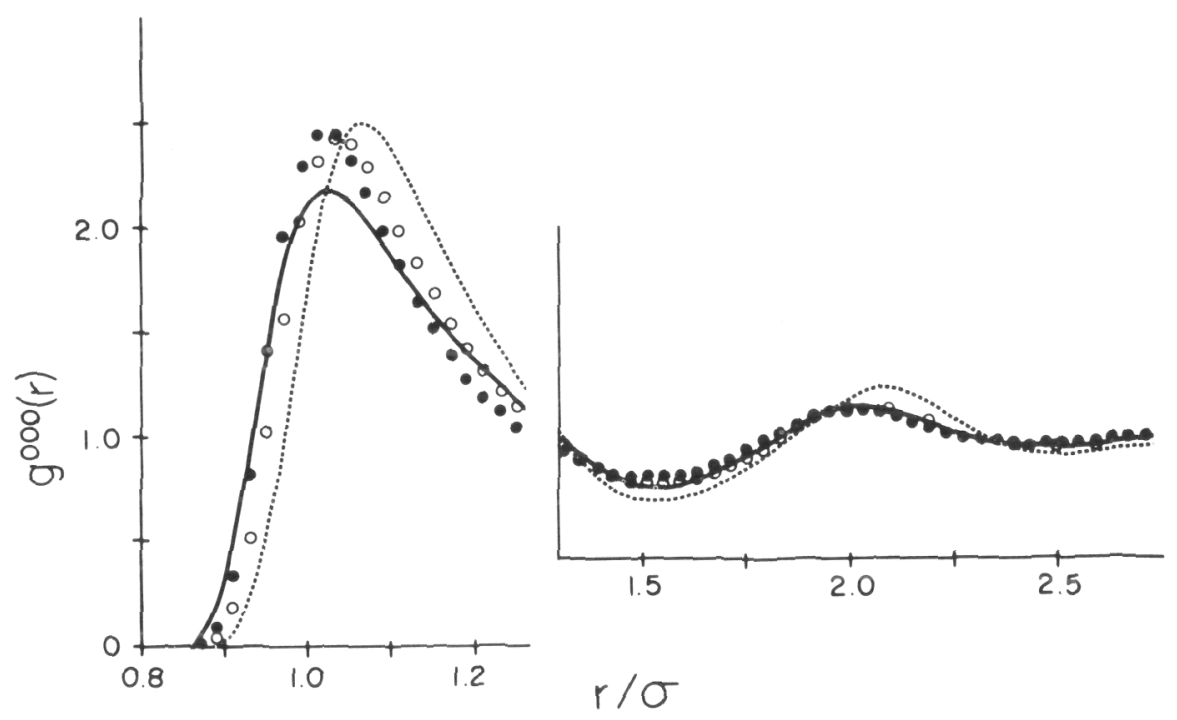

Figure 1. The radial distribution function $g^{000}(r)$ for fluids with $\rho^{*}=0 \cdot 8, \quad T^{*}=1 \cdot 35$, $\mu^{*}=1.5$ and $Q_{T}^{*}=0.72$. The solid dots, solid curve and dashed curve represent the MD, SCMF/QHNC and SCMF/LHNC (i.e. $g_{L J}(r)$ ) results, respectively, for the polarizable system with $\alpha^{*}=0.05$. The open circles are MD results for the nonpolarizable case (i.e. $\alpha^{*}=0$ ).

for both fluids the QHNC theory is reasonably accurate for $\beta P / \rho$ given that this quantity is very sensitive to relatively small errors in the positive and negative contributions to the pressure (cf. equation $(9 a)$ ).

The dielectric constants given by SCMF/QHNC and SCMF/LHNC theories are in very good agreement with the MD results. It can be seen from table $(a)$

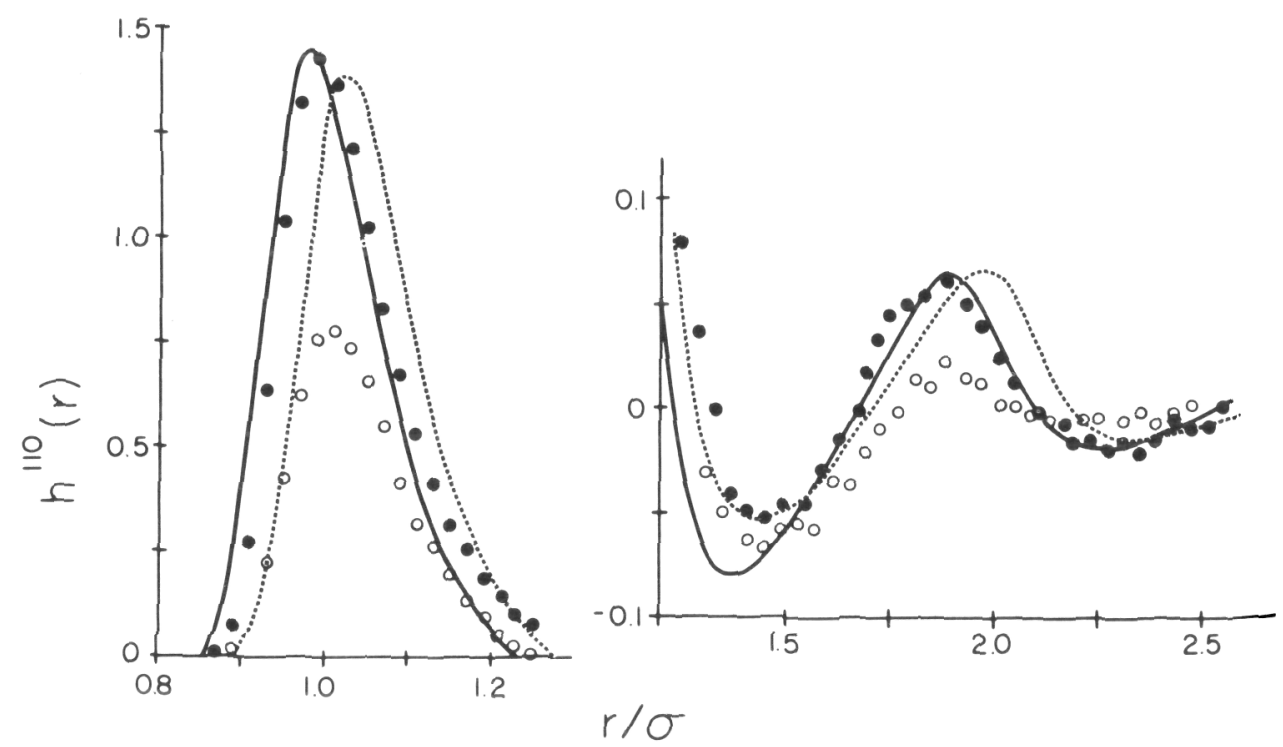

Figure 2. The coefficient $h^{110}(r)$. The parameters and curves are as in figure 1. 
that this is also true for the single particle properties. The results given by both the SCMF/QHNC and SCMF/LHNC theories for $m^{\prime}$ and $\alpha^{\prime}$ are in remarkably good agreement with the MD values.

(b) The pair distribution function

The (000), (110), (112), (121), (123), (220), (222) and (224) coefficients of the pair distribution function [cf. equation (10)] are plotted in figures 1-8. For the polarizable fluid MD, SCMF/QHNC and SCMF/LHNC results are shown. The MD results for the non-polarizable system are also included in the figures. The influence of polarizability is detectable for all projections but is particularly striking for $h^{110}(r)$ and $h^{112}(r)$ (cf. figures 2 and 3) which exhibit a large increase in the height of the first peak. This change in $h^{110}(r)$ must be an important factor contributing to the doubling of the dielectric constant discussed above. We also note (cf. figure 1) that the first peak in the radial distribution function, $g^{000}(r)=h^{000}(r)+1$, shifts to a smaller separation in response to the stronger electrostatic forces present in the polarizable fluid.

The agreement between the SCMF/QHNC theory and the MD calculations is generally good and is very similar to the agreement previously found [1] between MD and QHNC results for non-polarizable systems. It is clear from the figures that the influence of polarizability upon the pair correlation function is taken into account with reasonable accuracy by the SCMF/QHNC theory. In fact, since the agreement here is so similar to that observed for the non-polarizable case [1], it is

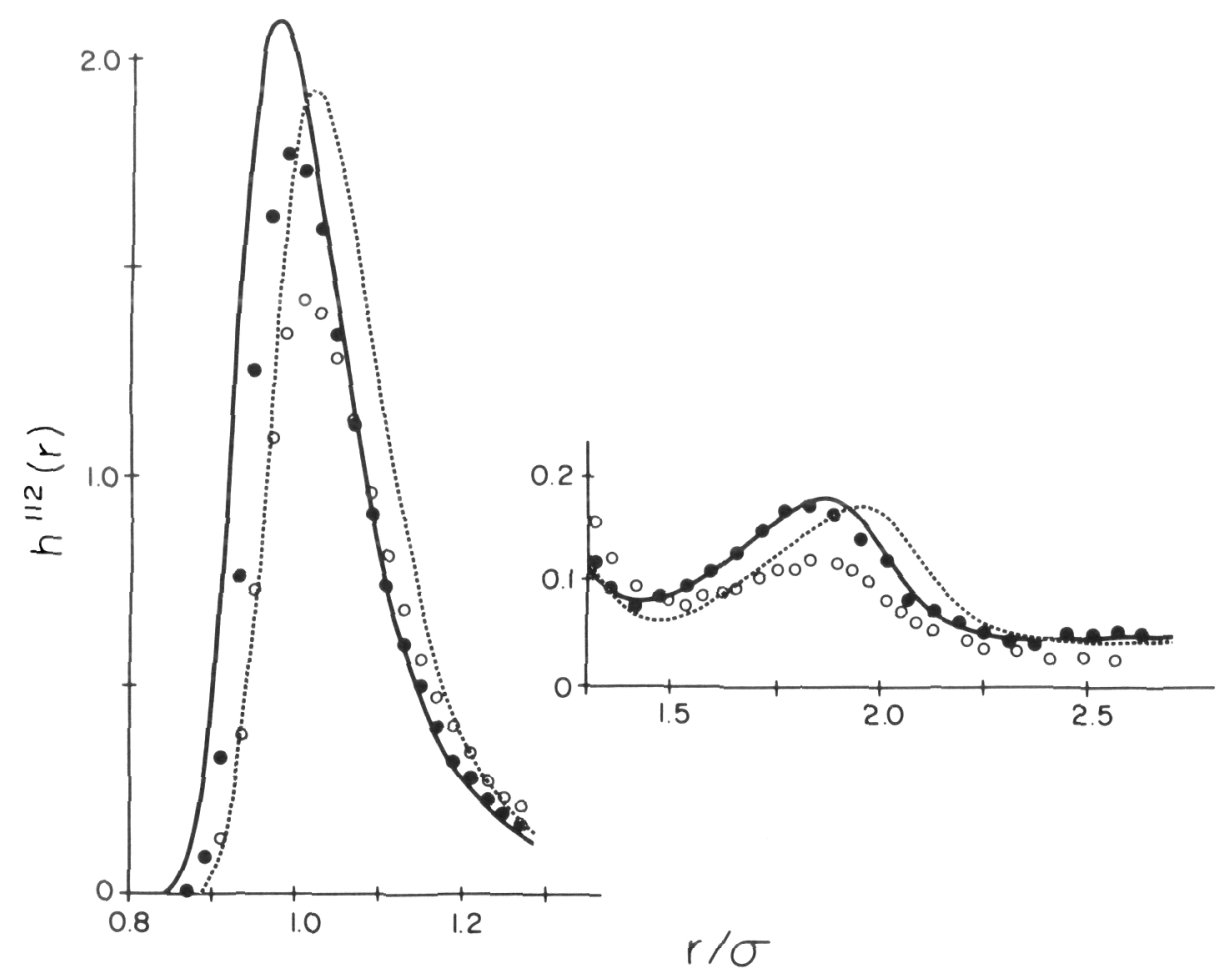

Figure 3. The coefficient $h^{112}(r)$. The parameters and curves are as in figure 1. 


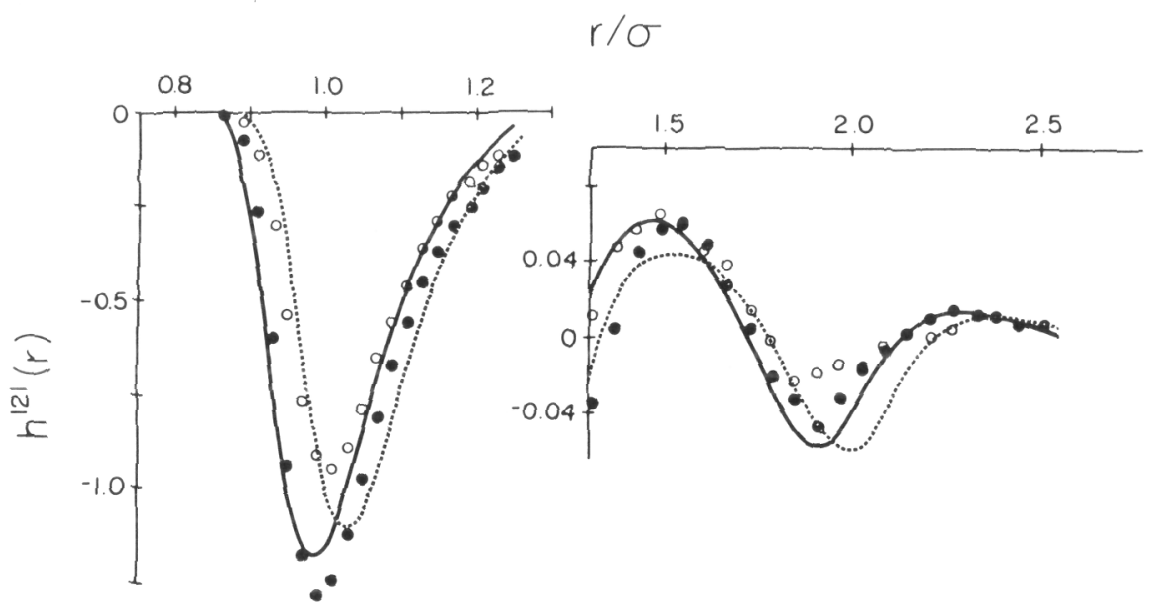

Figure 4. The coefficient $h^{121}(r)$. The parameters and curves are as in figure 1.

very likely that the discrepancies which do exist arise from the QHNC treatment of the effective system and not from the SCMF approximation.

It is also clear from figures $1-8$ that the $\mathrm{SCMF} / \mathrm{LHNC}$ results are generally less accurate than those obtained from the SCMF/QHNC theory. Again this is very similar to the non-polarizable case. The LHNC theory simply gives $g^{000}(r)=g_{\mathrm{LJ}}(r)$ and hence does not correctly take into account the shift in peak positions brought about by the electrostatic interactions.

\section{Summary and CONCLUSIONS}

In this paper we have reported MD results for both polarizable and nonpolarizable fluids with permanent dipole and quadrupole moments. The major purpose of this work was to provide a detailed test of the SCMF/QHNC and SCMF/LHNC theories [2] for polarizable fluids of this type. The MD calculations clearly show that for the present model molecular polarizability has an

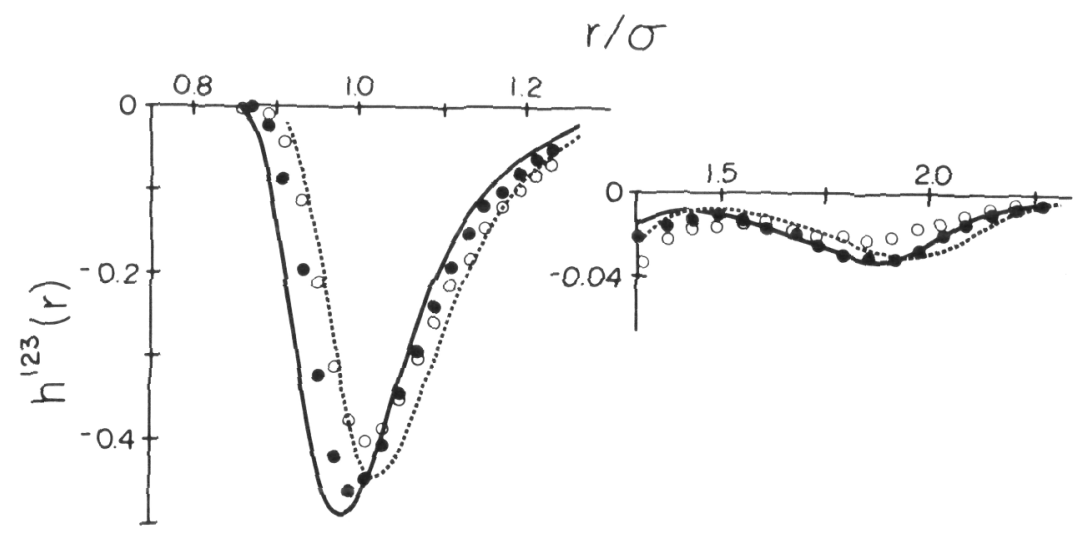

Figure 5. The coefficient $h^{123}(r)$. The parameters and curves are as in figure 1. 


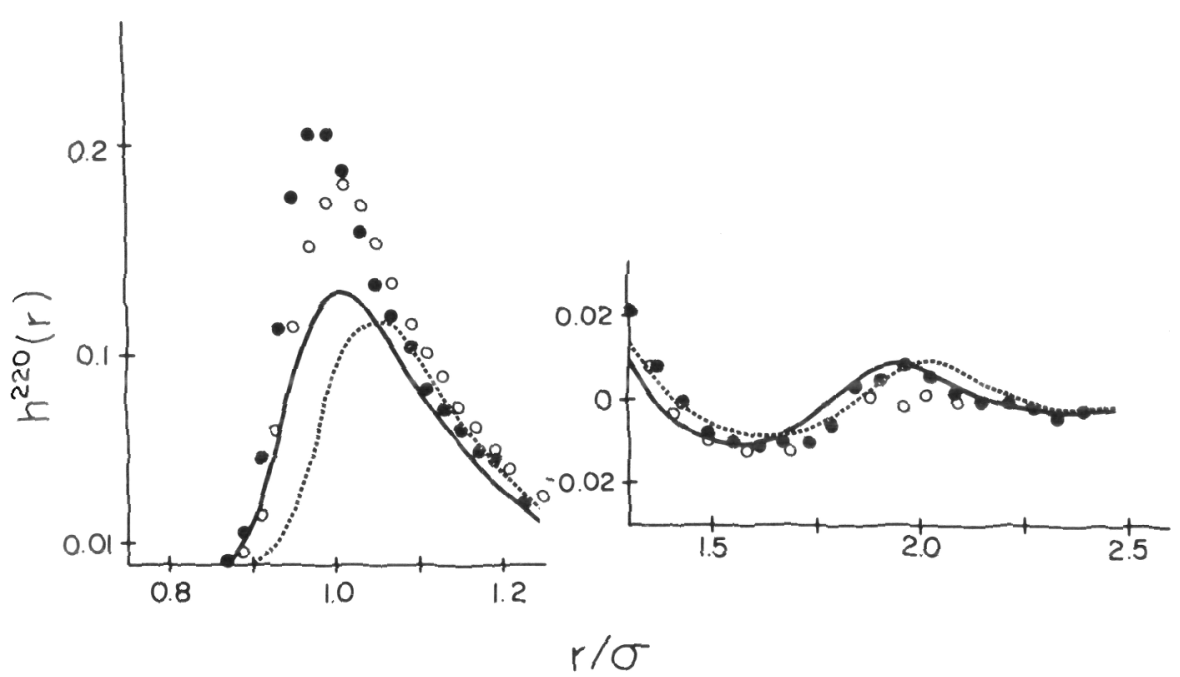

Figure 6. The coefficient $h^{220}(r)$. The parameters and curves are as in figure 1.

important influence upon the fluid properties leading, for example, to much larger dielectric constants than those obtained if polarizability is ignored. This is in accord with previous theoretical results [2].

It is shown that for some properties both the SCMF/QHNC theory and the SCMF/LHNC theory give a reasonably accurate description of the polarizable system. In particular the mean dipole moment and the dielectric constant given by these theories are in very good agreement with the MD results. The theoretical results for the thermodynamic quantities are somewhat less accurate with a discrepancy of $\sim 4$ per cent occurring in the total energy and much larger errors are evident in the compressibility factor.

The agreement between the MD and theoretical results for the coefficients of the pair correlation function is quite good for the SCMF/QHNC approximation but less accurate for the SCMF/LHNC theory. In fact the agreement between the MD and theoretical results for the polarizable fluid is very similar to that previously found [1] for non-polarizable systems. This leads us to the conclusion

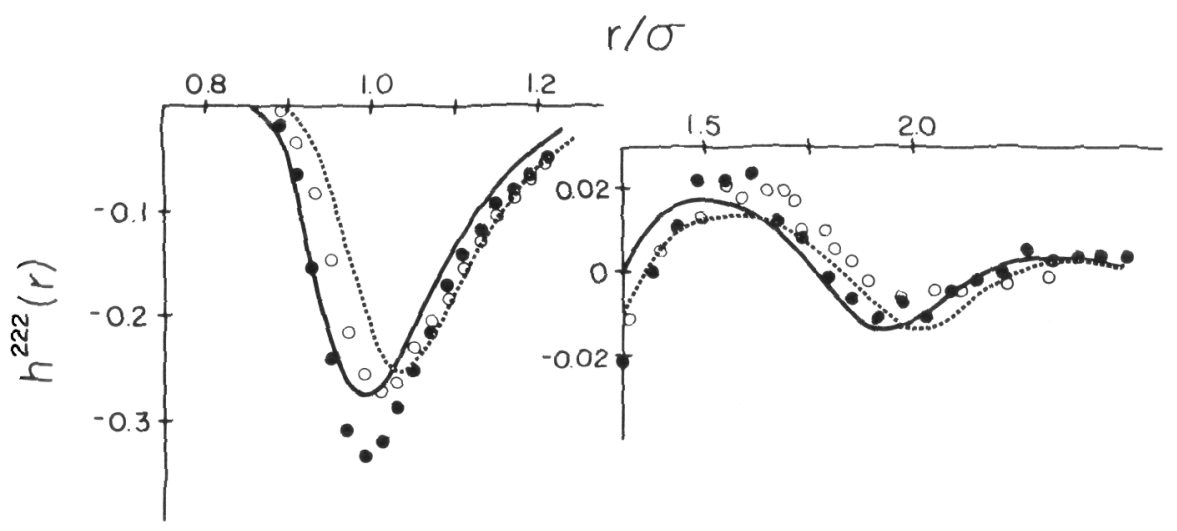

Figure 7. The coefficient $h^{222}(r)$. The parameters and curves are as in figure 1. 


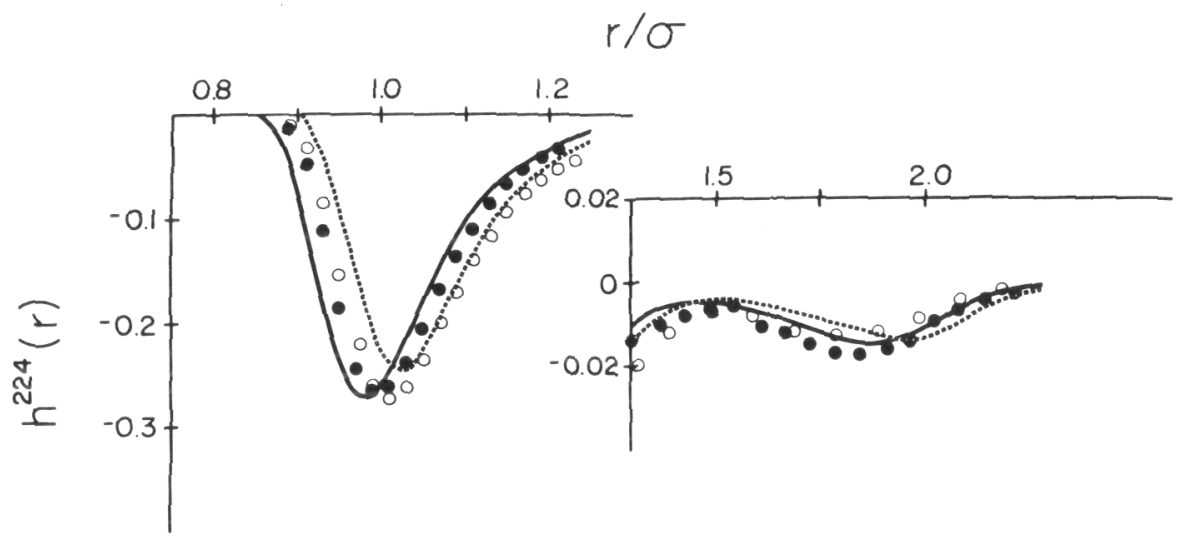

Figure 8. The coefficient $h^{224}(r)$. The parameters and curve are as in figure 1.

that most of the discrepancy between the MD and theoretical calculations for the polarizable case arises from the QHNC or LHNC treatment of the effective system rather than from the SCMF approximation.

One of us (J.J.W.) thanks the CNRS/NRCC for financial support under their cooperative research agreement. Also PGK and GNP gratefully acknowledge the financial support of the Natural Sciences and Engineering Research Council of Canada.

\section{REFERENCES}

[1] Levesque, D., Weis, J. J., and Patey, G. N., 1984, Molec. Phys., 51, 333.

[2] Carnie, S. L., and Patey, G. N., 1982, Molec. Phys., 47, 1129.

[3] Wertheim, M. S., 1973, Molec. Phys., 25, 211; 1973, Ibid., 26, 1425; 1977, Ibid., 33, 95; 1977, Ibid., 34, 1109.

[4] Venkatasubramanian, V., Gubbins, K. E., Gray, C. G., and Joslin, C. G., 1984, Molec. Phys., 52, 1411.

[5] Vesely, F. J., 1977, Y. comput. Phys., 24, 361.

[6] Pollock, E. L., Alder, B. J., and Patey, G. N., 1981, Physica A, 108, 14. Pollock, E. L., and Alder, B. J., 1980, Physica A, 102, 1.

[7] Stillinger, F. H., 1979, F. chem. Phys., 71, 1647.

[8] Barnes, P., Finney, J. L., Nicholas, J. D., and Quinn, J. E., 1979, Nature, Lond. $282,459$.

[9] Perer, P., Lee, W. K., and Prolofsky, E. W., 1983, F. chem. Phys., 79, 388.

[10] Murad, S., 1984, Molec. Phys., 51, 525.

[11] Neumann, M., and Steinhauser, O., 1983, Chem. Phys. Lett, 95, 417.

[12] Neumann, M., 1983, Molec. Phys., 50, 841.

[13] De Leeuw, S. W., Perram, J. W., and Smith, E. R., 1983, Proc. R. Soc. A, 388, 177.

[14] Alder, B. J., Weis, J. J., and Strauss, H. L., 1973, Phys. Rev. A, 7, 281. Alder, B J., Strauss, H. L., and Weis, J. J., 1973, F. chem. Phys., 59, 1002; 1975, Ibid., 62 2328.

[15] Verlet, L., and Weis, J. J., 1972, Phys. Rev. A, 5, 939. 